\title{
Downregulation of SWI5 and CTC1 genes: hepatitis B virus DNA polymerase transactivated protein 1-mediated inhibition of DNA repair
}

\author{
X.-K. YAO ${ }^{1,2 \&}$, Z.-P. PAN ${ }^{1,22}$, Y. LI ${ }^{3}$, Y.-Z. LUN $^{1,2 *}$, Q. CHI², S.-J. JIANG ${ }^{4}$, F. WANG ${ }^{2}$, W. SUI ${ }^{2}$
}

\begin{abstract}
${ }^{1}$ Pharmaceutical and Medical Technology College, Putian University, Putian 351100, P. R. China; ${ }^{2}$ Liaoning Provincial University Key Laboratory of Biophysics, College of Medicine, Dalian University, Dalian 116622, P. R. China; ${ }^{3}$ Department of pathology, Beijing Ditan Hospital, Capital Medical University, Beijing 100015, P. R. China; ${ }^{4}$ Department of Gynecology and Obstetrics, Affiliated Zhongshan Hospital of Dalian University, Dalian 116001, P. R. China
\end{abstract}

\begin{abstract}
Summary. - Hepatitis B virus (HBV) DNA polymerase transactivated protein 1 (HBVDNAPTP1) is a novel protein upregulated by HBV DNA polymerase, which has been screened by suppression subtractive hybridization technique (SSH) (GenBank Acc. No. AY450389). A vector pcDNA3.1 (-)/myc-His A-HBVDNAPTP1 was constructed and used to transfect acute monocytic leukemia cell line THP-1. HBVDNAPTP1 expression was detected by Western blot analysis in the cells. A cDNA library of genes downregulated by HBVDNAPTP1 in THP- 1 cells was made in pGEM-T Easy using SSH. The cDNAs were sequenced and analyzed with BLAST search against the sequences in GenBank. Some sequences, such as DNA repair protein SWI5 homolog (SWI5) and CTS telomere maintenance complex component 1 (CTC1), might be involved in DNA repair. Protein expression of SWI5 and CTC1 was identified by Western blot in THP-1 cells. HBVDNAPTP1 could downregulate the expression of SWI5 and CTC1 at translation level.
\end{abstract}

Keywords: hepatitis B virus; DNA polymerase; trans-regulation; suppression subtractive hybridization; SWI5; CTC1

\section{Introduction}

It is well known that, after the infection of target cells by $\mathrm{HBV}$, the interaction of the virus genome and proteins with genes and proteins in the target cells plays important roles in determining HBV replication, immune evasion, and chronic infection (Eyre et al., 2009). In recent years it has been found that a complex trans-regulation mechanism is involved in the interaction of HBV with the target cells,

*Corresponding author. E-mail: lunyz@163.com; phone: +86-41187402740. \&Equal contributors.

Abbreviations: $\mathrm{HBV}=$ hepatitis B virus; $\mathrm{SSH}=$ suppression subtractive hybridization; SWI5 $=$ DNA repair protein SWI5 homolog; SFR1 = SWI5-dependent homologous recombination repair protein 1 ; CTC $1=$ CTS telomere maintenance complex component 1; PILRA = paired immunoglobin-like type 2 receptor alpha; CIP4 $=$ Cdc42-interacting protein 4 ; $\mathrm{PBMC}=$ peripheral blood mononuclear cell and that the HBV proteins play a trans-regulative role in the gene expression in the target cells. The critical antigen components of HBV associated with the transregulation function have been identified in the target cells and the specific mechanisms have been clarified, which are of great significance in confirming the pathogenic mechanisms of HBV and discovering effective prevention and treatment methods. HBV DNA polymerase transactivated protein 1 (HBVDNAPTP1) is a protein, which is worth studying. Therefore, SSH technology has been used to study the target genes of the HBV DNA polymerase, which was verified by dot blot hybridization. The HepG2 hepatoblastoma cell line was screened to obtain a novel gene (GenBank Acc. No. AY450389), which was located on the long arm of chromosome 9, region 2, band 2, sub-band 31 (9q22.31). Using the Unigene database for the expression analysis of tissue distribution, this gene was shown to be expressed in a variety of tissues, but not in the pituitary gland, tonsil, tongue, thymus, trachea or umbilical cord. A preliminary 
study clarified that HBVDNAPTP1 is localized in the cytoplasm (Lun et al., 2014a).

In the present study, we first analyzed the expression of HBVDNAPTP1 in THP-1 cells and subsequently screened genes transactivated by HBVDNAPTP1 using SSH. SSH was designed to generate a cDNA library, which is enriched in differentially expressed sequences and, more importantly, equalized for the number of individual cDNA species, thus allowing the detection of rare transcripts (Ghorbel and Murphy, 2011). The full-length gene from the library was searched for homologs in GenBank. Finally, the relationship of the SWI5 and CTC1 and the HBVDNAPTP1 is discussed. SWI5 is a component of the SWI5-SFR1 complex, which is required for double-strand break repair via homologous recombination (Akamatsu and Jasin, 2010). CTC1 is a component of the CST complex, which binds to single-stranded DNA and is required to protect telomeres from DNA degradation (Miyake et al., 2009).

\section{Materials and Methods}

Construction of vectors. For construction of eukaryotic expression vector pcDNA3.1 (-)/myc-His A-HBVDNAPTP1, the HBVDNAPTP1 fragment was PCR-amplified with the forward primer (5'- GGATCCATGATGTTTGTGCT-GCTAAAC), containing a BamHI site, and reverse primer (5'-AAGCTTATAAGTC CTCTCTAAAATTGC), containing a HindIII site. The fragment was inserted into the cloning vector pGEM-T (Promega), resulting in pGEM-T-HBVDNAPTP1. A BamHI-HindIII fragment was isolated from the vector and inserted into BamHI and HindIII digested pcDNA3.1 (-)/myc-His A (Invitrogen), giving pcDNA3.1 $(-) /$ myc-His A-HBVDNAPTP1. The vector was sequenced and digested with the corresponding restriction enzymes to confirm the sequence accuracy.

Cell culture and transient transfection. THP-1 cells were cultivated in Dulbecco's modified Eagle's medium (DMEM, Invitrogen) containing $100 \mathrm{IU}$ of penicillin and $100 \mu \mathrm{g}$ of streptomycin per ml, supplemented with $10 \%(\mathrm{v} / \mathrm{v})$ heat-inactivated fetal bovine serum (FBS, Hyclone), at $37^{\circ} \mathrm{C}$ in $5 \% \mathrm{CO}_{2}$ and with $90 \%$ relative humidity. The cells were seeded one day prior to transfection at a density of $8 \times 10^{5}$ cells per $35 \mathrm{~mm}$ dish, and reached $50 \%$ confluence at the time of transfection. All transfections were performed with FuGENE6 Transfection Reagent (Roche) according to the manufacturer's instructions. The medium was changed $5 \mathrm{hr}$ after transfection, and the cells were harvested $40-48 \mathrm{hr}$ after transfection. All transfections and assays were repeated independently three times in triplicate.

Detection of HBVDNAPTP1 expression. mRNA from THP-1 cells transfected with pcDNA3.1 (-)/myc-His A-HBVDNAPTP1 or pcDNA3.1(-)/myc-His A was isolated using a micro mRNA purification kit (Amersham Biosciences), and cDNAs were reverse-transcribed from the mRNA. HBVDNAPTP1 expression was detected by Western blot assay using the lysates of the THP-1 cells. The extracts were boiled for $5 \mathrm{~min}$ and separated on SDS-PAGE, then transferred to the nitrocellulose membrane (Pierce). The membranes were incubated with anit-myc monoclonal antibody and HRP-labeled goat anti-mouse IgG as the first and second antibodies, respectively, then with a SuperSignal West Pico Chemiluminescent Substrate Working Solution (Pierce) according to the manufacturer's instructions. The immunoreactive bands were visualized after exposure to X-ray film.

Generation and analysis of a subtracted cDNA library. SSH was performed using the PCR-Select ${ }^{\mathrm{TM}} \mathrm{cDNA}$ subtraction kit (Clontech) according to the manufacture's protocol. In brief, $2.0 \mu \mathrm{g}$ of polyA+ mRNA, from each the pcDNA3.1 (-)/myc-His A-HBVDNAPTP1 driver group and the pcDNA3.1 (-)/myc-His A tester group was subjected to CDNA synthesis. After restriction digestion with RsaI, small fragments of cDNAs were obtained. The tester cDNAs were then subdivided into two parts, and ligated with the specific adaptors 1 and 2, respectively. After two subtractive hybridization reactions and two suppression PCR amplifications, differentially expressed cDNAs were selectively amplified. Then the second PCR products were used as templates for PCR amplification of G3PDH (a housekeeping gene) at 18, 23, 28 and 33 cycles, respectively, to analyze subtraction efficiency. The second PCR products were directly purified using the Wizard PCR-Preps DNA Purification System (Promega), and inserted into pGEM-T Easy (Promega) to construct the subtracted library. Colony PCRs were conducted to confirm the size of cDNA inserts ranging between 200 and 1000 bp by using T7/SP6-specific primers localized in pGEM-T Easy. After the DNA sequencing of the positive colonies, nucleotide homology searches were performed using the BLAST program at NCBI.

Detection the effects of HBVDNAPTP1 on SWI5 and CTC1 expression. To detect the effects of HBVDNAPTP1 on SWI5 and CTC1 protein, total soluble proteins were extracted from the transfected THP-1 cells and separated on 12.5\% SDS-PAGE for Western blot assay. The expression of SWI5 and CTC1 was probed by monoclonal antibodies (Santa Cruz), and antibody against $\beta$-actin (Santa Cruz) was used as an internal reference. The immunoreactive bands were visualized in an UVP Biospectrum Imaging System. The remaining steps are essentially the same as those described in Detection of HBVDNAPTP1 expression.

\section{Results}

\section{Transient expression of HBVDNAPTP1 in THP-1 cells}

Digestion of recombinant vector pcDNA3.1 (-)/mycHis A-HBVDNAPTP1 with BamHI/HindIII and BglII yielded expected bands (Fig. 1). The DNA sequencing results indicated that the recombinant vector containing the HBVDNAPTP1 sequence was completely correct. The HBVDNAPTP1 proteins in the THP-1 cells were successfully detected by Western blot (Fig. 2). 


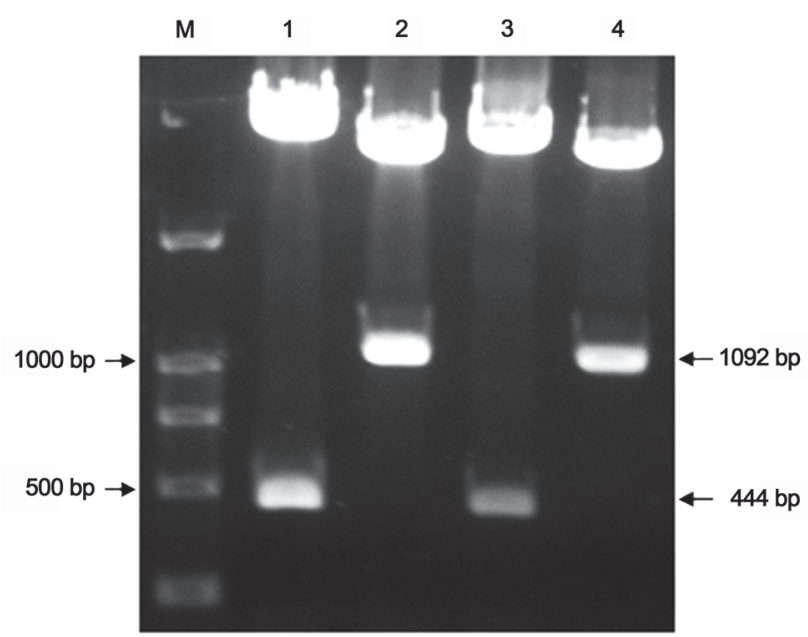

Fig. 1

Digestion Identification of pcDNA3.1(-)/myc-His A-HBVDNAPTP1 DNA Marker DL2,000 (lane M). pcDNA3.1(-)/myc-His A-HBVDNAPTP1 digested by BamHI and HindIII (lane 1 and lane 3). pcDNA3.1(-)/myc-His A-HBVDNAPTP1 digested by BgIII (lane 2 and lane 4 ).

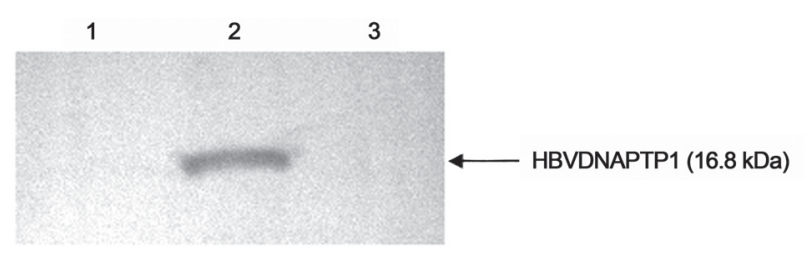

Fig. 2

Western blot analysis of transient expression of HBVDNAPTP1 in THP-1 cells

Lysates from THP-1 cells (lane 1), lysates from THP-1 cells transfected with pcDNA3.1(-)/myc-His A-HBVDNAPTP1 (lane 2), lysates from THP-1 cells transfected with pcDNA3.1(-)/myc-His A (lane 3).

Analysis of the cDNA subtracted library down transregulated by HBVDNAPTP1

To gain a general view of genes, which may be involved in the pathogenesis of $\mathrm{HBV}$, we identified downregulated genes in THP-1 cells expressing HBVDNAPTP1 by the generation of a subtracted cDNA library. Subtraction efficiency analysis showed that the PCR products of the housekeeping gene G3PDH in the unsubtracted library were obviously visible after 18 cycles; however, 28 cycles were required in the subtracted one (Fig. 3), indicating that the abundance of non-differentially expressed gene was effectively reduced and that the subtraction method had high subtraction efficiency. Using SSH, a total of 100 positive clones were obtained.

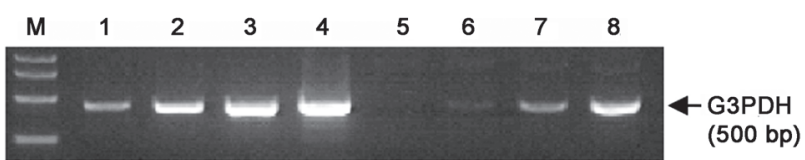

Fig. 3

Analysis of subtracted cDNA library

Reduction of G3PDH abundance showed high subtraction efficiency. Unsubtracted secondary PCR product (lanes 1-4) and subtracted PCR product (lanes 5-8). DNA Marker DL2,000 (TaKaRa) (lane M). 18 cycles (lanes 1, 5). 23 cycles (lanes 2,6 ). 28 cycles (lanes 3,7 ). 33 cycles (lanes 4,8 ).

These clones were prescreened by using PCR amplification to ensure that they had different inserts before sequencing. Among these clones, 79 contained inserts of 200 to $1000 \mathrm{bp}$.

Table 1. Sequence analysis of 32 clones isolated from subtracted cDNA library downregulated by HBVDNAPTP1

\begin{tabular}{|c|c|c|}
\hline Gene name & $\begin{array}{l}\text { GenBank } \\
\text { Acc. No. }\end{array}$ & $\begin{array}{l}\text { Homology } \\
(\%)\end{array}$ \\
\hline \multicolumn{3}{|l|}{ Cell signal transduction and apoptosis } \\
\hline CDC42-interacting protein 4 (TRIP10) & NM_004240 & 100 \\
\hline FGFR1 oncogene partner 2 (FGFR1OP2) & NM_015633 & 99 \\
\hline $\begin{array}{l}\text { Guanine nucleotide binding protein, beta } \\
\text { polypeptide } 2 \text { (GNB2) }\end{array}$ & NM_005273 & 100 \\
\hline $\begin{array}{l}\text { Insulin-like growth factor binding protein } \\
3 \text { (IGFBP3) }\end{array}$ & NM_001013398 & 100 \\
\hline Prothymosin, alpha (PTMA) & NM_002823 & 100 \\
\hline Thymosin beta $4, \mathrm{X}$-linked (TMSB4X) & NM_021109 & 99 \\
\hline \multicolumn{3}{|l|}{ Cell energy and substance metabolism } \\
\hline $\begin{array}{l}\text { ATPase, } \mathrm{Na}+/ \mathrm{K}+\text { transport, beta } 3 \\
\text { polypeptide (ATP1B3) }\end{array}$ & NM_001679 & 99 \\
\hline $\begin{array}{l}\text { ATP synthase, } \mathrm{H}+\text { transport, mitochon- } \\
\text { drial } \mathrm{F}_{\mathrm{o}} \text { complex, subunit F6 (ATP5J) }\end{array}$ & NM_001003701 & 99 \\
\hline $\begin{array}{l}\text { DnaJ (Hsp40) homolog, subfamily C, } \\
\text { member } 8 \text { (DNAJC8) }\end{array}$ & NM_014280 & 99 \\
\hline Lactate dehydrogenase B (LDHB) & NM_001174097 & 98 \\
\hline $\begin{array}{l}\text { NADH dehydrogenase subunit } 5 \text { (mito- } \\
\text { chondrion) }\end{array}$ & NC_012920 & 100 \\
\hline Transferrin receptor (TFRC) & NM_003234 & 99 \\
\hline \multicolumn{3}{|l|}{ Cell transcription and protein synthesis } \\
\hline $\begin{array}{l}\text { Eukaryotic translation initiation factor } 3 \text {, } \\
\text { subunit K (EIF3K) }\end{array}$ & NM_013234 & 88 \\
\hline H2A histone family, member Z (H2AFZ) & NM_002106 & 100 \\
\hline $\begin{array}{l}\text { NADH dehydrogenase } 1 \text { alpha subcom- } \\
\text { plex, } 4,9 \mathrm{kDa} \text { (NDUFA4) }\end{array}$ & NM_002489 & 98 \\
\hline $\begin{array}{l}\text { RNA Polymerase II (DNA-directed) } \\
\text { polypeptide L (POLR2L) }\end{array}$ & NM_021128 & 100 \\
\hline \multicolumn{3}{|l|}{ Cell DNA damage and repair } \\
\hline $\begin{array}{l}\text { DNA repair protein SWI5 homolog } \\
\text { (SWI5) }\end{array}$ & NM_001040011 & 100 \\
\hline $\begin{array}{l}\text { CTS telomere maintenance complex } \\
\text { component } 1 \text { (CTC1) }\end{array}$ & NM_025099 & 100 \\
\hline
\end{tabular}




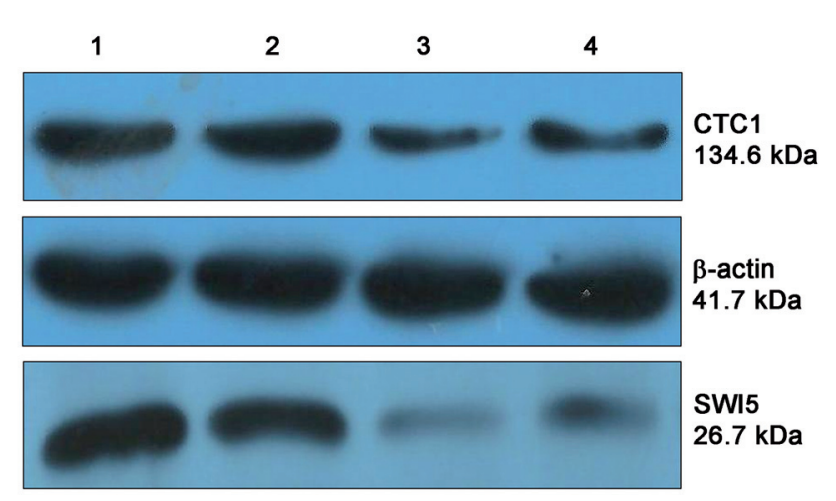

Fig. 4

Western blot analysis of SWI5 and CTC1 expression in THP-1 cells THP-1 cells transfected with pcDNA3.1(-)/myc-His A (lane 1,2). THP-1 cells transfected with pcDNA3.1(-)/myc-His A-HBVDNAPTP1 (lane 3, 4).

A total of 32 clones from the cDNA library were randomly chosen and sequenced, and their nucleotide sequence homology searches were performed using the BLAST program at NCBI. The analysis results showed that there were 18 coding sequences, all of which were known. These genes with known functions can be divided into four groups, namely genes related to cell signal transduction and apoptosis, cell energy and substance metabolism, cell transcription and protein synthesis, and DNA damage and repair. Interestingly, in the final group, the cellular proteins SWI5 and CTC1 were downregulated by HBVDNAPTP1. A summary of the data is presented in Table 1 .

\section{HBVDNAPTP1 downregulated the SWI5 and CTC1} expression

In order to further elucidate the mechanisms of HBVDNAPTP1 on the SWI5 and CTC1 expression at the translation level, we investigated the effects of HBVDNAPTP1 on the expressions of the genes. The signals were normalized using the housekeeping gene G3PDH. As shown in Fig. 4, the SWI5 and CTC1 protein levels significantly decreased after transient transfection with pcDNA3.1 (-)/myc-His A-HBVDNAPTP1 (Student-Neumann-Keuls' test, $\left.\mathrm{n}=3,{ }^{\star} \mathrm{P}<0.05\right)$. The Western blot analysis indicated that the expression of the gene was high in the control group, whereas its expression in the experimental group was significantly lowered. The results indicated that HBVDNAPTP1 could downregulate the expressions of SWI5 and CTC1 at the translation level.

\section{Discussion}

Based on previous research, HBVDNAPTP1 interacts with PILRA intracellular domain. PILRA is a transmem- brane receptor with the effect of inhibitory regulation, which is not expressed in lymphocytes but mostly in monocytes (Fournier et al., 2000). As PILRA binds with its ligand (primary cytokines), it triggers PILRA to terminate cell proliferation signals or induce apoptosis signal (Saha et al., 2010; Xiao et al., 2011). It is worth mentioning that in normal PBMCs PILRA binds with cytokines but does not terminate cell proliferation or trigger apoptosis (Hou et al., 2005). Due to the interaction of HBVDNAPTP1 and PILRA, it is easy to speculate that HBVDNAPTP1 is very likely to bind with PILRA to mediate the negative regulation of the JAK/STAT signaling pathway, and thus activate the monocyte apoptosis signal (Lun et al., 2014a). In addition, it was shown that the expression of CIP4 at both the transcription and translation levels in the experimental group, which stably expressed $\mathrm{HB}$ VDNAPTP1, were lower than those of the controls (Lun et al., 2014b). CIP4 serves as a scaffolding protein for CDC42, a member of the F-BAR family, which regulates membrane deformation and endocytosis, thus playing a key role in extracellular matrix deposition and cell migration (Bai et al., 2012). In vitro studies have confirmed that inhibiting CIP4 expression significantly reduced cell invasiveness and migration (Koshkina et al., 2013).

Chronic infection with HBV is still a large health problem worldwide. The liver is the main target organ of HBV infection, but many extrahepatic tissues and organs, including the heart, spleen, lung, kidney etc., are also infected by HBV (Pontisso et al., 2008; Chotiyaputta et al., 2010; Lai and Liaw, 2010). In recent years, various forms of HBV DNA, replicative RNA intermediates and antigen components have been detected in the PBMC of chronically HBV-infected individuals (Lu et al., 2009). The major clinical effect of HBV infection of PBMC is host immune dysfunction, which leads to chronic HBV infection and causes latent infection, chronic infection and mother-to-child transmission. Moreover, reinfection may occur after liver transplantation or use of drugs to clear virus from the serum (Coppola et al., 2008; Gatta et al., 2008). Due to the fact that HBV DNA can be replicated and transcribed by integration into the PBMC genome, the PBMCs have become important replication sites and propagation vectors for HBV (Hollinger and Sood, 2010). PBMCs are mainly composed of lymphocytes, monocytes, granulocytes and other immunocompetent cells, which are believed to play a crucial role in the body's immune response against $\mathrm{HBV}$ infection. However, HBV infection with $\mathrm{PB}-$ MCs leads to cell dysfunction and a decline in the number of PBMCs, which indicates that HBV DNA replication and transcription in PBMCs cause cell apoptosis and inhibition of cell proliferation. Due to the inadequate $\mathrm{HBV}$-specific cellular immune response, the removal of virus is difficult (Hollinger and Sood, 2010; Bai et al., 2011).

To gain further insight on information about the genes regulated by HBVDNAPTP1, SSH was used to clone the 
genes downregulated by HBVDNAPTP1. Excitingly, the downregulation of SWI5 and CTC1 expression was observed. SWI5 can form a stable complex with SFR1 in vitro and in vivo. The C-terminal Swi5 domain of SWI5 and the middle coiled-coil region of SFR1 dictate this conserved interaction. The depletion of SWI5 or SFR1 from human cells causes defects in homologous recombination repair. Human SWI5-SFR1 has an evolutionarily conserved function in homologous recombination repair (Yuan and Chen, 2011). CTC1 is a component of the CST complex, a complex, which binds to single-stranded DNA and is required to protect telomeres from DNA degradation. The CST complex binds single-stranded DNA with high affinity in a sequenceindependent manner, while isolated subunits bind DNA with low affinity by themselves. In addition to telomere protection, the CST complex likely has a more general role in DNA metabolism at non-telomeric sites (Surovtseva et al., 2009). The low expression of SWI5 and CTC1 has been implicated in the PBMCs behavior of patients with chronic hepatitis $\mathrm{B}$. The above results coincide with the results of previous research. The biologic significance of SWI5 and CTC1 gene down-regulation by HBVDNAPTP1, however, has not been confirmed.

To further elucidate the regulatory mechanisms of HBVDNAPTP1 on SWI5 and CTC1 expression, the downregulated expression of SWI5 and CTC1 in THP-1 cells has been confirmed at the protein level by transient transfection. It is reasonable to believe that the transformation effect of HBVDNAPTP1 is involved in the downregulation of the expression of SWI5 and CTC1. Therefore, HBVDNAPTP1 may be involved in the positive regulation of monocyte apoptosis initiation. These findings provide new insight on the biological functions of HBVDNAPTP1, and new directions to elucidate the mechanisms of chronic infection with HBV.

Acknowledgement. The authors thank the technical staff of the Department of Biotechnology, Beijing Centre for Physical and Chemical Analysis, Beijing, P. R. China, for providing excellent technical assistance.

\section{References}

Akamatsu Y, Jasin M (2010): Role for the mammalian Swi5-Sfr1 complex in DNA strand break repair through homologous recombination. PLoS Genet. 6, e1001160. http://dx.doi. org/10.1371/journal.pgen.1001160

Bai GQ, Li SH, Yue YF, Shi L (2011): The study on role of peripheral blood mononuclear cell in HBV intrauterine infection. Arch. Gynecol. Obstet. 283, 317-321. http://dx.doi. org/10.1007/s00404-010-1366-8

Bai S, Zeng R, Zhou Q, Liao W, Zhang Y, Xu C, Han M, Pei G, Liu L, Liu X, Yao Y, Xu G (2012): Cdc42-interacting protein-4 promotes TGF-Beta1-induced epithelial-mesenchymal transition and extracellular matrix deposition in renal proximal tubular epithelial cells. Int. J. Bio.l Sci. 8, 859-869.

Chotiyaputta W, Pelletier SJ, Fontana RJ, Lok ASF (2010): Longterm efficacy of nucleoside monotherapy in preventing $\mathrm{HBV}$ infection in HBsAg-negative recipients of anti-HBcpositive donor livers. Hepatol. Int. 4, 707-715. http:// dx.doi.org/10.1007/s12072-010-9188-0

Coppola N, Pisapia R, Tonziello G, Martini S, Imparato M, Piai G, Stanzione M, Sognelli C, Filippini P, Piccinino F, Sagnelli E (2008): Virological pattern in plasma, peripheral blood mononuclear cells and liver tissue and clinical outcome in chronic hepatitis B and C virus coinfection. Antivir. Ther. 13, 307-318.

Eyre NS, Phillips RJ, Bowden S, Yip E, Dewar B, Locarnini SA, Beard MR (2009): Hepatitis B virus and hepatitis C virus interaction in Huh-7 cells. J. Hepatol. 51, 446-457. http:// dx.doi.org/10.1016/j.jhep.2009.04.025

Fournier N, Chalus L, Durand I, Garcia E, Pin JJ, Churakova T, Patel S, Zlot C, Gorman D, Zurawski S, Abrams J, Bates EE, Garrone P (2000): FDF03, a novel inhibitory receptor of the immunoglobulin superfamily, is expressed by human dendritic and myeloid cells. J. Immunol. (Baltimore, Md.: 1950) $165,1197-1209$.

Gatta A, Giannini C, Lampertico P, Pontisso P, Quarta S, Zignego AL, Atzeni F, Sarzi-Puttini P (2008): Hepatotropic viruses: new insights in pathogenesis and treatment. Clin. Exp. Rheumatol. 26, S33-S38.

Ghorbel MT, Murphy D (2011): Suppression subtractive hybridization. Methods Mol. Biol. (Clifton, N.J.) 789, 237-259. http://dx.doi.org/10.1007/978-1-61779-310-3_15

Hollinger FB, Sood G (2010): Occult hepatitis B virus infection: a covert operation. J. Viral Hepat. 17, 1-15. http://dx.doi. org/10.1111/j.1365-2893.2009.01245.x

Hou W, Liu KZ, Li MW, Wo JE (2005): Effect of IFNalpha-2a on Fas expression and apoptosis rate of peripheral blood cytotoxic T cells in patients with hepatitis B. Hepatobiliary Pancreat. Dis. Int. 4, 403-405.

Koshkina NV, Yang G, Kleinerman ES (2013): Inhibition of Cdc42Interacting Protein 4 (CIP4) Impairs Osteosarcoma Tumor Progression. Curr. Cancer Drug Targets 13, 48-56. http://dx.doi.org/10.2174/156800913804486593

Lai M, Liaw YF (2010): Chronic Hepatitis B: Past, Present, and Future. Clin. Liver Dis. 14, 531-546. http://dx.doi. org/10.1016/j.cld.2010.05.003

Lu L, Zhang HY, Yueng YH, Cheung KF, Luk JM, Wang FS, Lau GKK (2009): Intracellular levels of hepatitis B virus DNA and pregenomic RNA in peripheral blood mononuclear cells of chronically infected patients. J. Viral Hepat. 16, 104-112. http://dx.doi.org/10.1111/j.1365-2893 $.2008 .01054 . \mathrm{x}$

Lun YZ, Chi Q, Wang XL, Wang F, Sui W (2014a): Identification of paired immunoglobulin-like type 2 receptor alpha as hepatitis B virus DNA polymerase transactivated protein 1 interacting proteins. Mol. Med. Rep. 9, 720-724.

Lun YZ, Xu CB, Chi Q, Wang XL, Sui W, Jiang SJ (2014b): CDC42Interacting Protein 4 Gene Is Down Trans-Regulated by 
HBV DNA polymerase Trans Activated Protein 1. Iran. J. Public Health 43, 282-290.

Miyake Y, Nakamura M, Nabetani A, Shimamura S, Tamura M, Yonehara S, Saito M, Ishikawa, F (2009): RPA-like Mammalian Ctc1-Stn1-Ten1 Complex Binds to SingleStranded DNA and Protects Telomeres Independently of the Pot1 Pathway. Mol. Cell 36, 193-206. http://dx.doi. org/10.1016/j.molcel.2009.08.009

Pontisso P, Vidalino L, Quarta S, Gatta A (2008): Biological and clinical implications of HBV infection in peripheral blood mononuclear cells. Autoimmun. Rev. 8, 13-17. http:// dx.doi.org/10.1016/j.autrev.2008.07.016

Saha B, Prasanna SJ, Chandrasekar B, Nandi D (2010): Gene modulation and immunoregulatory roles of Interferon gamma. Cytokine 50, 1-14. http://dx.doi.org/10.1016/j. cyto.2009.11.021
Surovtseva YV, Churikov D, Boltz KA, Song X, Lamb JC, Warrington R, Leehy K, Heacock M, Price CM, Shippen DE (2009): Conserved telomere maintenance component 1 interacts with STN1 and maintains chromosome ends in higher eukaryotes. Mol. Cell 36, 207-218. http://dx.doi. org/10.1016/j.molcel.2009.09.017

Xiao W, Kashiwakura J, Hong H, Yasudo H, Ando T, MaedaYamamoto M, Wu D, Kawakami Y, Kawakami T (2011): Phospholipase C-beta3 regulates FcvarepsilonRImediated mast cell activation by recruiting the protein phosphatase SHP-1. Immunity 34, 893-904. http://dx.doi. org/10.1016/j.immuni.2011.04.010

Yuan JS, Chen JJ (2011): The Role of the Human SWI5-MEI5 Complex in Homologous Recombination Repair. J. Biol. Chem. 286, 9888-9893. http://dx.doi.org/10.1074/jbc. M110.207290 